\title{
Paste Pumping with Centrifugal Pumps: Evaluation of the Hydraulic Institute Chart De-Rating Procedures
}

\author{
G. Sery, B. Kabamba, P. Slatter Flow Process Research Centre, Cape Peninsula University of Technology, \\ South Africa
}

\section{INTRODUCTION}

Walker and Goulas have suggested that the Hydraulic Institute Chart, primarily meant for centrifugal pump performance prediction for Newtonian materials with a known viscosity, be extended to non-Newtonian slurries. The performance correction must make use of an apparent viscosity calculated at a shear rate of $2 \mathrm{~N}$ ( $N$ being the impeller angular speed) for low flow rates, and for high flow rates at a shear rate between 100$1500 \mathrm{~s}^{-1}$. This is considered ambiguous. The present work calculates an apparent viscosity from a pump impeller average shear rate determined using the Metzner and Otto method and evaluates the accuracy of the Hydraulic Institute Chart in pump performance predictions for non-Newtonian materials.

\section{LITERATURE REVIEW}

It is impossible to determine a given centrifugal pump performance curves by calculation because of the many design and manufacturing parameters (blade angle, gap width, surface roughness, etc). Centrifugal pump performance curves, therefore, are always established experimentally by actual measurement, with water as the standard test liquid. In many cases, however, the liquid pumped (such as slurries) is more viscous. In these cases, pump performance differs considerably from that when pumping water. Viscous liquids cause more hydraulic losses in the pump, so that at higher viscosities, pumping head and pump efficiency decrease while required power increases. The pumping head and pump efficiency curves for viscous liquids fall below the corresponding water performance curves, but the shut-off head point remains the same, regardless of viscosity.

Figure 1 shows how pump performance is affected by increased mixture volumetric concentration $\left(\mathrm{C}_{\mathrm{v}}\right)$.

Procedures for de-rating centrifugal pumps from their water performance characteristics when pumping slurries are empirical.

Figure 2 shows the Hydraulic Institute correction chart for determining the performance of a conventional centrifugal pump handling a viscous liquid, based on known water characteristics. 


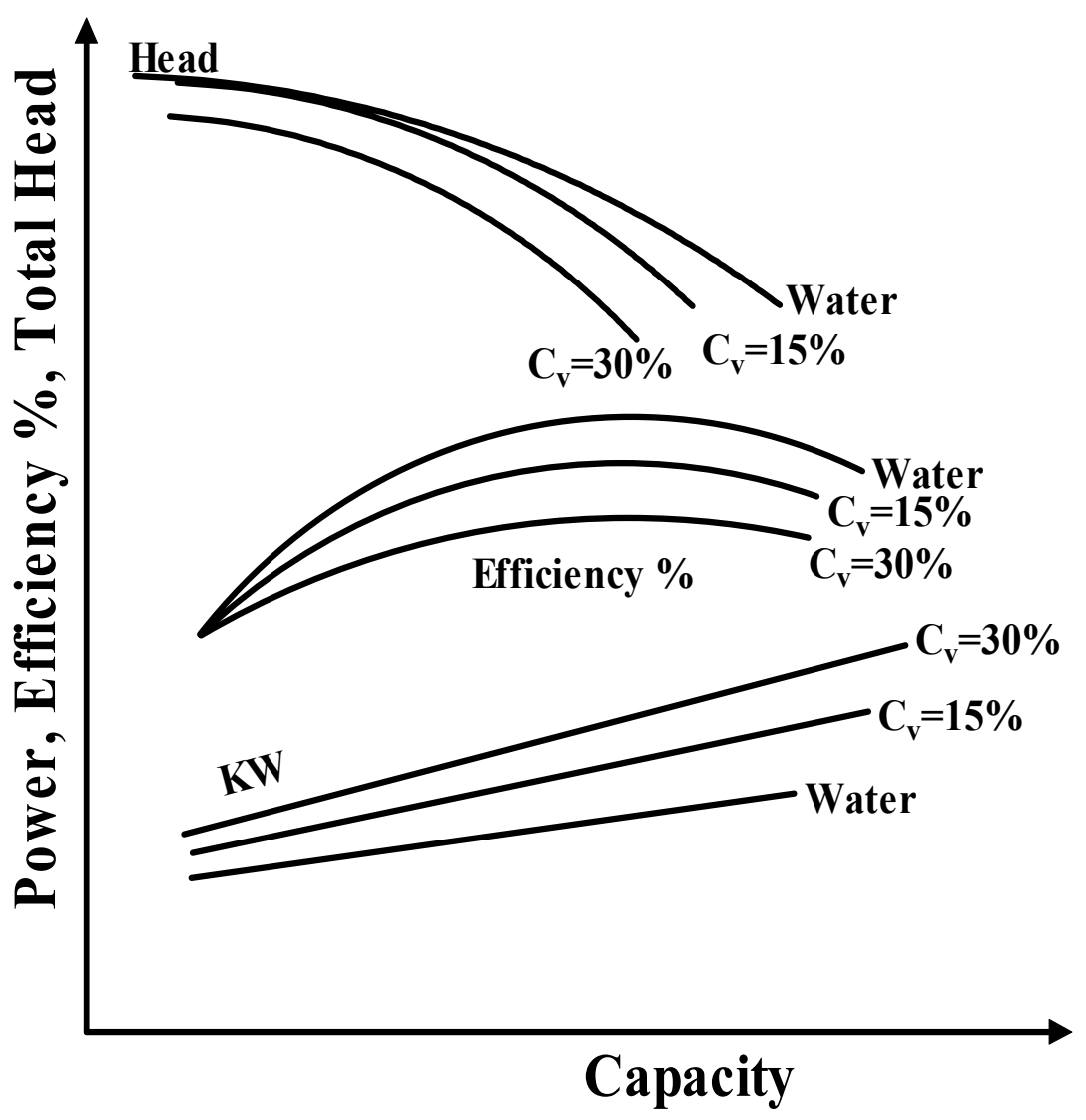

Figure 1 Pump performance for non-Newtonian slurry (Angle and Crisswell, 1997)

The Hydraulics Institute model uses two diagrams to correct for liquid viscosity. The first employs the capacity (pumping rate) at the best efficiency point (bep) of the water performance curves. The diagram parameters are the pumping head at bep and the kinematic viscosity $v$ (defined as the ratio of the dynamic or shear viscosity to the material density) of the liquid pumped. Based on these values, $C_{h}, C_{q}$ and $C_{\eta}$, the correction factors for the head, capacity and efficiency respectively, are determined. Four different $C_{h}$ values are needed to obtain several points on the most important performance curve, pumping head vs. pump capacity $(\mathrm{Q})$. These $\mathrm{C}_{\mathrm{h}}$ factors are determined for the following capacities: $0.6 \mathrm{Q}_{\text {bep }}, 0.8 \mathrm{Q}_{\text {bep }}$, $\mathrm{Q}_{\text {bep }}$ and $1.2 \mathrm{Q}_{\text {bep }}$. We can easily plot the corrected performance curves, valid for the viscous liquid, from the calculated heads and efficiencies as a function of the corrected pump capacities. Accuracy is ensured because four different points on each performance curve are known, and, the head vs. capacity curve has an additional set point corresponding to the shut-off head at zero pumping rate. 


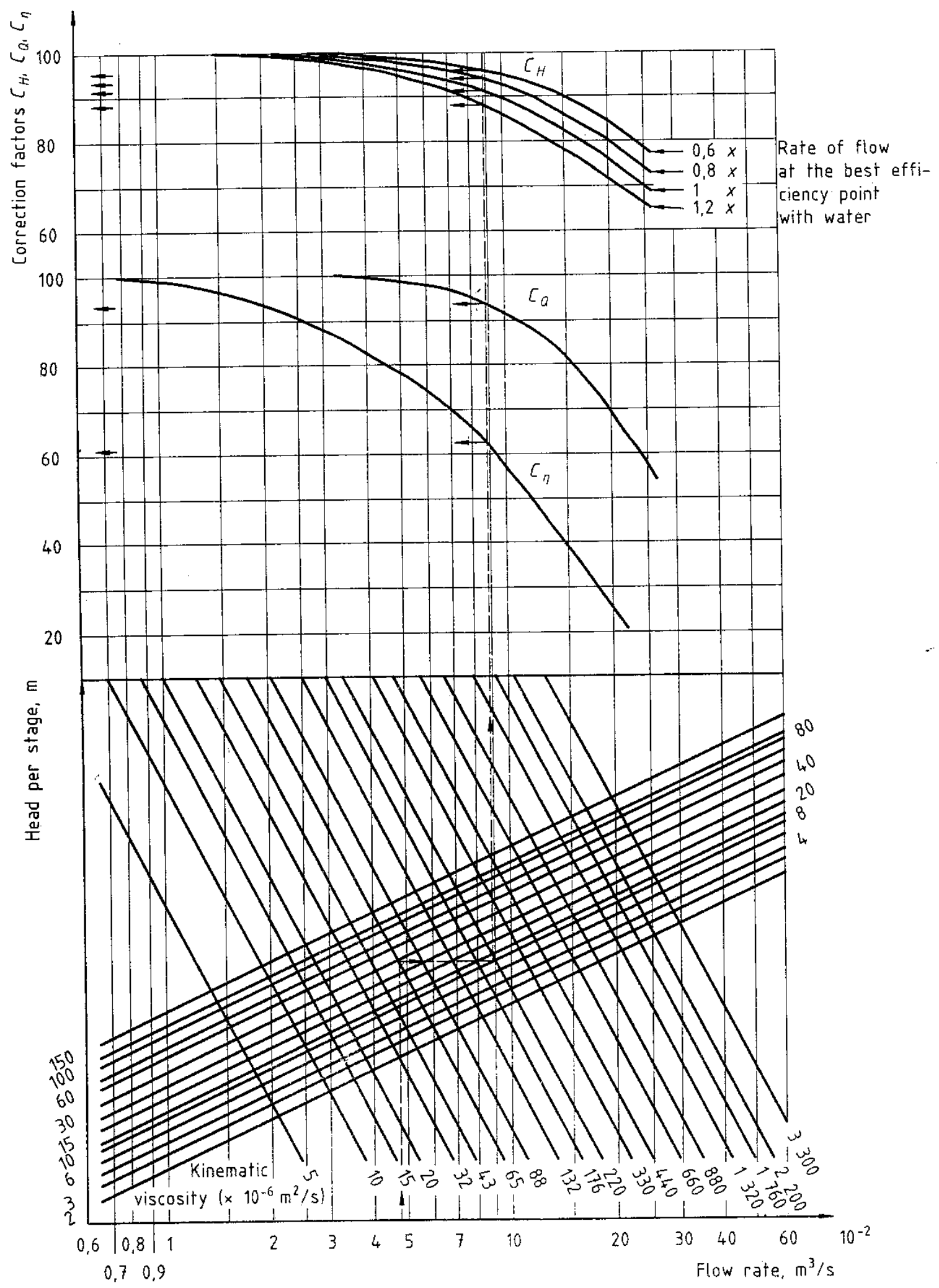

Figure 2 Hydraulic Institute Charts (International Standard 9906, 1999) 
Walker and Goulas (1984) have investigated the performance prediction of centrifugal pumps for nonNewtonian slurries based on the Hydraulic Institute Charts. For high flow rates, the kinematic viscosity was calculated using the plastic viscosity and the slurry density. The plastic viscosity is determined at the highest shear rate possible from the rheological results (generally between 100-1500 s-1) because, at increased flow rates, turbulence is greater and consequently shear rates should also be greater. For low flow rates, an apparent viscosity has to be determined for shear rates corresponding to $2 N$, where $N$ is the impeller angular speed. They claimed that at low shear rates the apparent viscosity is most sensitive to the influence of the yield stress which appeared to be the dominating factor in the head reduction. Their experimental data was within $\pm 5 \%$ of the predicted values.

Although not explicitly mentioned, the existence of a boundary shear rate is advocated. Slatter (2005) confirmed that the boundary shear rate marks the limit between yield stress and plastic viscosity domination of viscosity. However, it is suggested that the calculation of a plastic viscosity for shear rate values between 100-1500 s $\mathrm{s}^{-1}$ makes the procedure of Walker and Goulas a guess-work or at best a procedure which is valid only when experimental data are available which is contrary to the objective of predicting the pump performance. Furthermore, the plastic viscosity is regarded by the research community as a fitting parameter of the Bingham plastic model with no rheological meaning. It is not applicable to any rheological model.

The objective of the work presented here is to determine a characteristic pump impeller average shear rate, using the Metzner and Otto (1957) method, in order to calculate an apparent viscosity based on the fluid's rheology. The point of departure for the current work is the Hydraulic Institute Charts.

\section{EXPERIMENTAL TEST RIG AND APPARATUS}

The Flow Process Research Centre test rig is shown schematically in Figure 3. A closed 5 vane (type AH) $6 / 4$ centrifugal pump was used in the test work. The impeller is metal lined with a diameter of $365 \mathrm{~mm}$. Pump tests were run using two kaolin slurry concentrations of $21 \%$ and $17 \%$ by volume, at pump speeds of 1400 and $1100 \mathrm{rpm}$. Pump suction and discharge pressures were measured with point pressure transducers. Pipe pressure gradient was measured with differential pressure transducers and flow rate by means of a magnetic flow meter. A speed-torque unit was fitted between the motor and the pump. Instruments outputs were stored to computer via an HP data acquisition unit. 


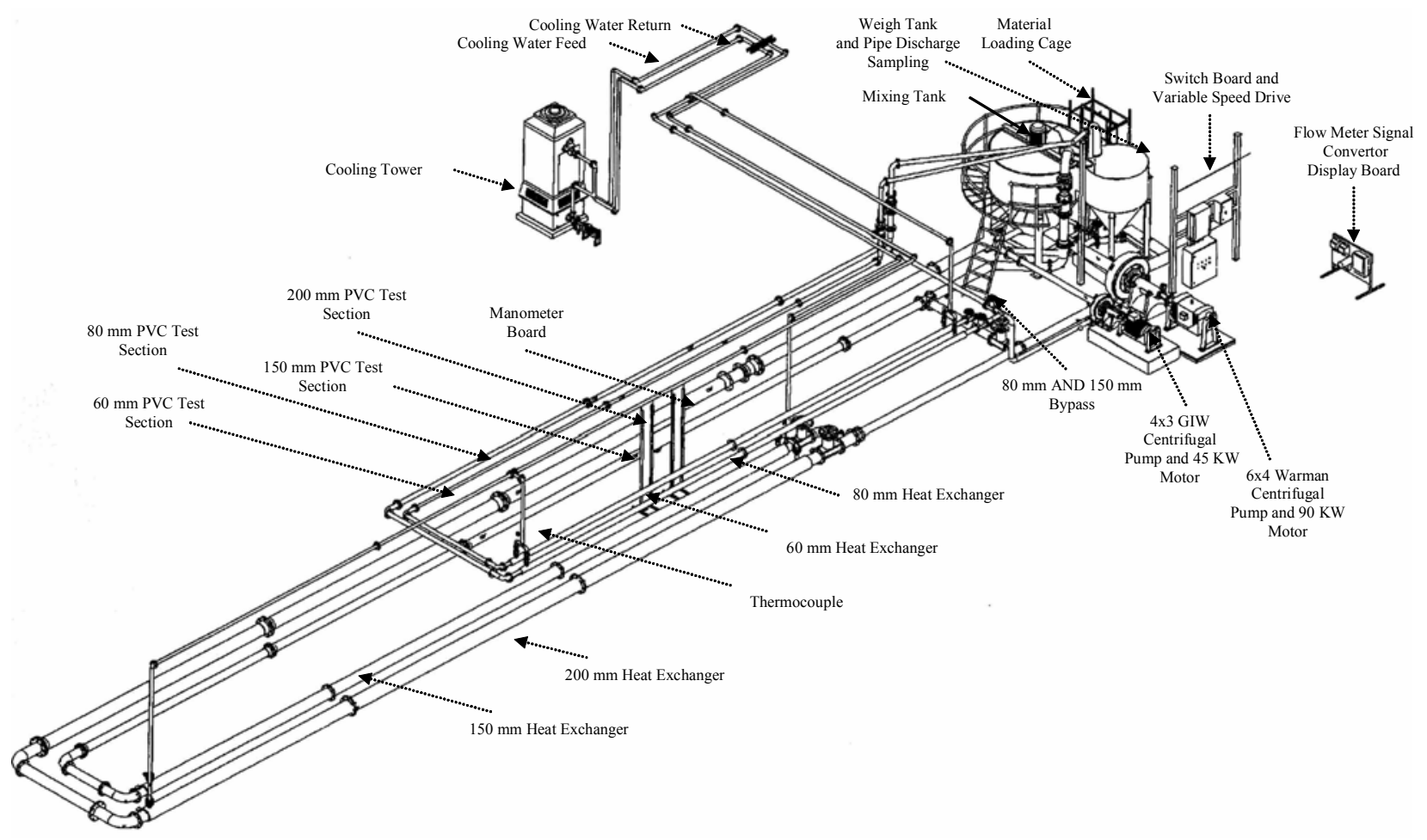

Figure 3 Schematic of test rig at the Flow Process Research Centre

\section{APPARENT VISCOSITY CALCULATION}

Metzner and Otto studied the mixing of shear thinning fluids in the laminar regime. They postulated a relationship between what they called the impeller average shear rate and $N$ given by Equation 1.

$$
\dot{\gamma}_{a}=K_{S} N
$$

where $K_{s}$ is a constant of proportionality which has to be found experimentally for each impeller, as it depends on the system geometry. It can be determined by deriving power correlations for Newtonian and non-Newtonian fluids. For a Newtonian fluid, the relationship is given by Equation 2.

$$
K_{p}=N_{p} \operatorname{Re}
$$

where

$$
N_{p}=\frac{P}{\rho N^{3} D^{5}}
$$

and

$$
\operatorname{Re}=\frac{\rho N D^{2}}{\mu}
$$


are the power number and the Reynolds number respectively. In the laminar regime, $K_{p}$ is a constant; $P, \rho, D$ and $\mu$ are power, density, impeller diameter and fluid viscosity respectively.

The dimensionless groups defined for non-Newtonian fluids are represented by a family of parallel curves, each corresponding to a particular value of $n$, where $n$ is the flow behaviour index of a rheological model (Rieger and Novak, 1973). Equation 2 can therefore be generalised as:

$$
K_{p}=N_{p} \operatorname{Re}_{g e n}
$$

For non-Newtonian materials, $K_{p}(n)$ has the same meaning as $K_{p}$.

Based on the yield pseudo-plastic rheological model, the general Reynolds number $\operatorname{Re}_{g e n}$ is expressed as:

$$
\operatorname{Re}_{\text {gen }}=\frac{\rho N D^{2}}{\frac{\tau_{y}}{N}+K(N)^{n-1}}
$$

where $\tau_{y}$ and $K$ are fluid yield stress and fluid consistency index respectively.

Rieger and Novak also pointed out that when a classical dimensionless representation of power data $\left(N_{p}\right.$ vs $\mathrm{Re}$ ) is desired, then $K_{s}$ values are needed. The general Reynolds number expression then becomes:

$$
\operatorname{Re}_{\text {gen }} 2=\frac{\rho N D^{2}}{\frac{\tau_{y}}{K_{s} N}+K\left(K_{s} N\right)^{n-1}}
$$

Furthermore, Brito-De la Fuente et al.(1992) demonstrated that if the Rieger and Novak method (Equation 7) is compared with the Metzner and Otto method, then:

$$
\frac{K_{p}(n)}{K_{p}}=\frac{1}{K_{S}}\left(\frac{\tau_{y}+K\left(K_{S} N\right)^{n}}{\tau_{y}+K(N)^{n}}\right)
$$

Equation 8 enables the calculation of $K_{s}$ once $K_{p}, K_{p}(\mathrm{n})$ and the fluid's rheological parameters are known. Normally $K_{p}$ is found experimentally through $N_{p}$ vs Re test work using Newtonian materials. In this study, $K_{p}$ was calculated with a non-linear regression algorithm proposed by Brito-De la Fuente et al. (1997), as given in Equation 9.

$$
K_{p}(n)=a b^{(n-1)} c^{(n-1) / n}
$$

In Equation 9 the parameter "a" is equal to the Newtonian power constant, $K_{p}$.

Several non-Newtonian materials were used to determine the impeller shear rate. The rheological parameters and the derived $K_{p}(n)$ and $K_{s}$ values for the 6/4 pump are presented in Table 1. 
Table 1

Non-Newtonian fluids rheological parameters with derived $K_{p}(n)$ and $K_{s}$ values

\begin{tabular}{|l|l|l|l|l|l|l|}
\hline Material & $\rho\left(\mathbf{K g} / \mathbf{m}^{\mathbf{3}}\right)$ & Yield Stress $\mathbf{( P a )}$ & $\mathbf{K}\left(\mathbf{P a} . \mathbf{s}^{\mathbf{n}}\right)$ & $\mathbf{n}$ & $\boldsymbol{K}_{\boldsymbol{p}}(\mathbf{n})$ & $\boldsymbol{K}_{\boldsymbol{s}}$ \\
\hline Bentonite 7.34\% w/w & 1046.4 & 7.04 & 0.01 & 1 & 54.51 & 1.15 \\
\hline CMC 6\% w/w & 1032.3 & 0 & 1.95 & 0.66 & 40.32 & 3.56 \\
\hline Kaolin 17\% & 1284 & 88 & 7.26 & 0.30 & 5.44 & 14.02 \\
\hline Kaolin 21\% & 1351.1 & 201 & 5.91 & 0.36 & 6.22 & 11.14 \\
\hline
\end{tabular}

Figure 4 shows that when the Rieger and Novak method is followed, the classical dimensionless representation of power data ( $N_{p}$ vs Re) is achieved.

In Table 2, apparent viscosity values used in the Hydraulic Institute Charts are presented. They were calculated as.

$$
\eta_{\text {app }}=\frac{\tau_{y}}{K_{S} N}+K\left(K_{S} N\right)^{n-1}
$$

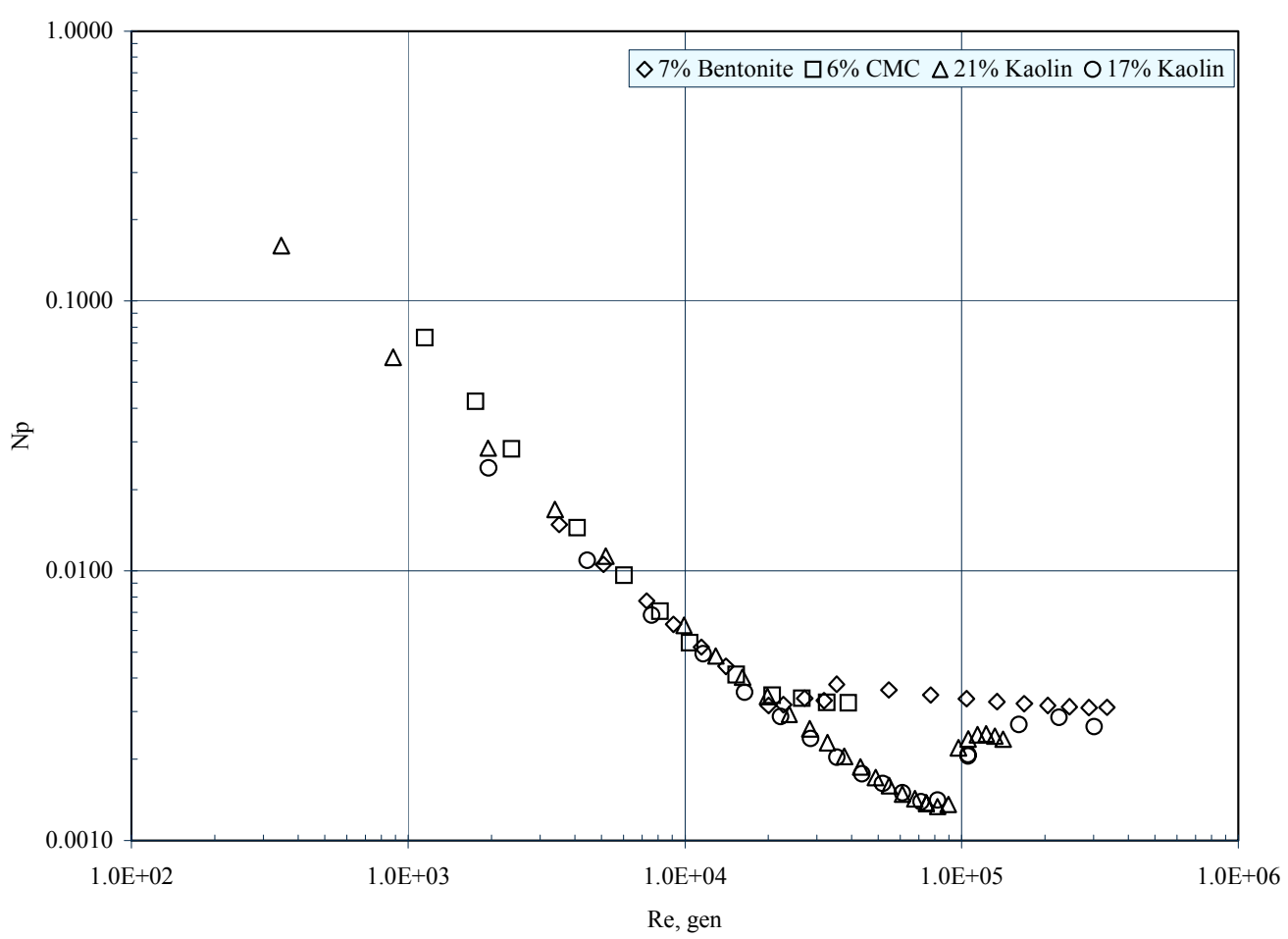

Figure 4 Dimensionless representation of $N_{p}$ vs $\operatorname{Re}$ for the $6 / 4$ pump

Table 2 Apparent viscosity calculated from Equation 10

\begin{tabular}{|l|l|l|}
\hline \multirow{2}{*}{ Pump rpm } & \multicolumn{2}{|c|}{ Apparent viscosity (Pa.s) } \\
\cline { 2 - 3 } & \multicolumn{2}{|c|}{ Material } \\
\cline { 2 - 3 } & Kaolin 17\% & Kaolin 21\% \\
\hline 1100 & 0.096 & 0.217 \\
\hline 1200 & 0.089 & 0.201 \\
\hline 1300 & 0.083 & 0.187 \\
\hline 1400 & 0.078 & 0.185 \\
\hline
\end{tabular}




\section{PUMP PREDICTION RESULTS}

Figures 5 and 6 present both the experimental data and the prediction results for 1100 and $1400 \mathrm{rpm}$.
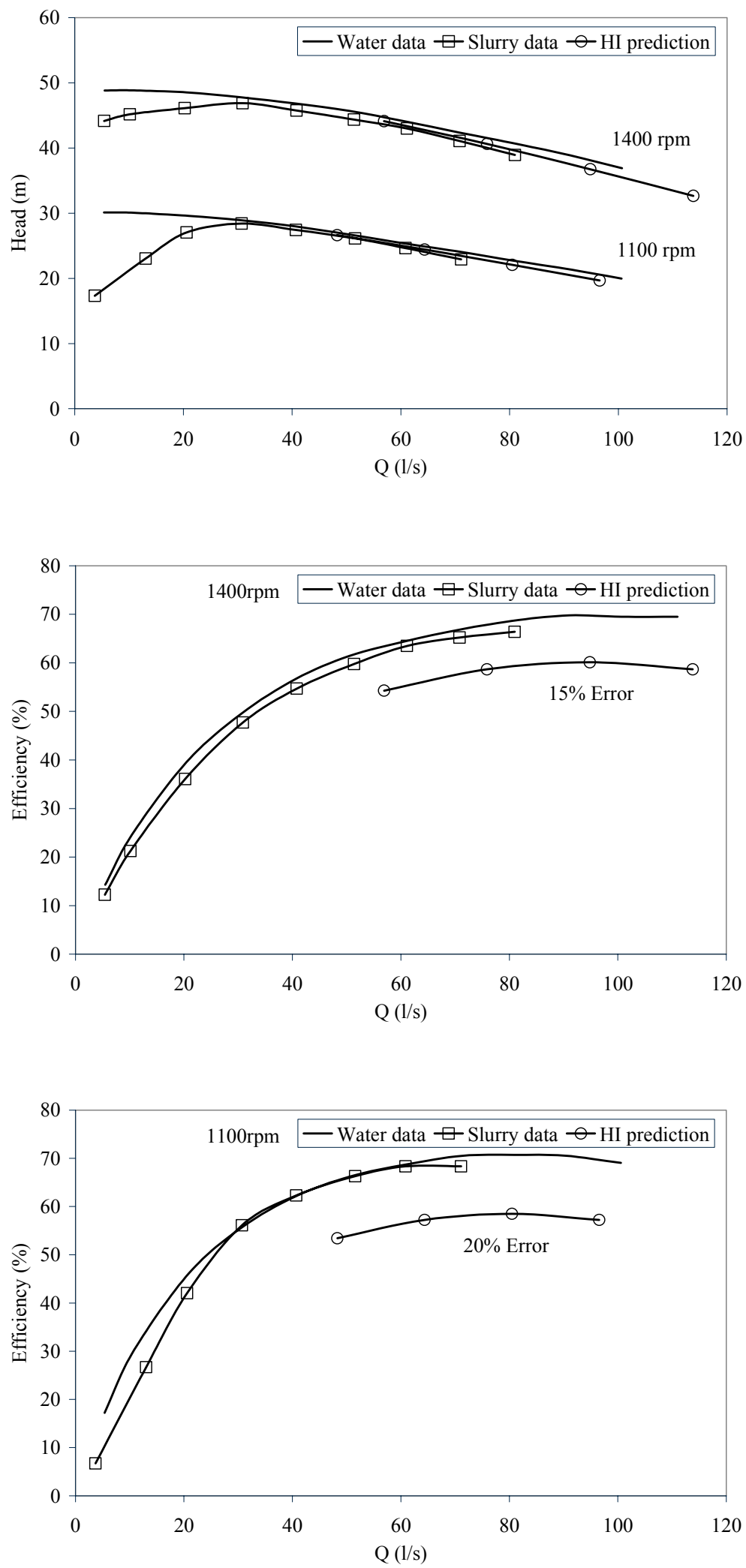

Figure 5 Experimental and predicted results for $17 \%$ kaolin 

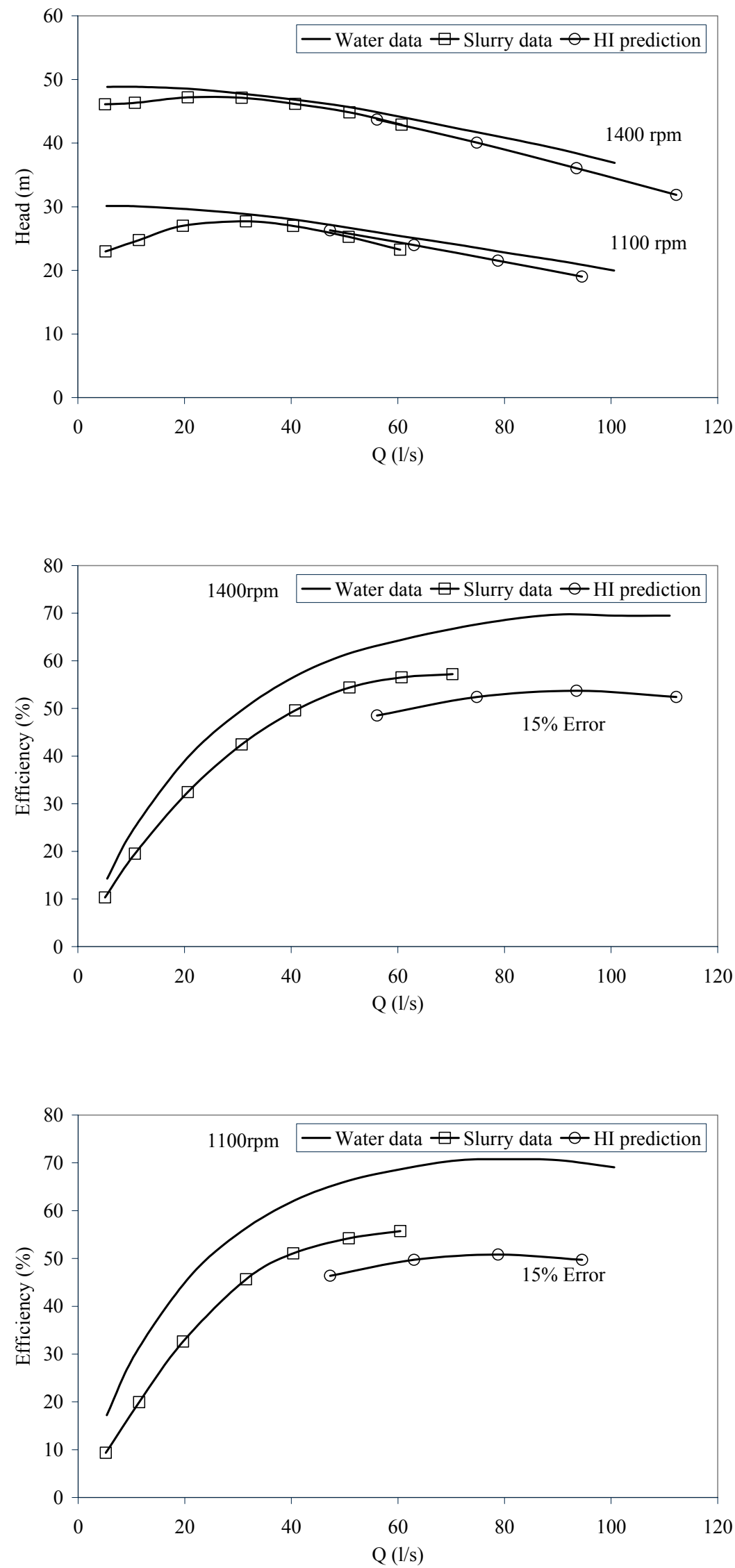

Figure 6 Experimental and predicted results for $21 \%$ kaolin 


\section{CONCLUSION}

The performance prediction of a 6 x 4 centrifugal pump based on the HI Chart was examined.

The apparent viscosity was calculated from the Metzner and Otto method. Pump experiments were conducted with $17 \%$ and $21 \%$ kaolin slurries. Test data was compared with predicted values from the Hydraulic Institute Charts and it is suggested that as far as the head is concerned, the modified Metzner and Otto method could yield results with a reasonable accuracy (less than 5\%). However, in the 60\% to $120 \%$ bep and where data is available the error in predicted efficiency was as high as $20 \%$. It is also observed that the error margin is decreased as the fluid becomes more viscous. A plausible explanation for this is that Equation 1 applies only when viscous forces dominate the process under investigation (laminar regime) and would underestimate the power requirement when a turbulent flow regime prevails. This raises the concept of a boundary shear rate or critical Reynolds number which perhaps needs to be defined.

The work presented here is a preliminary investigation into the application of the Metzner and Otto concept to centrifugal pump performance prediction. It is worthy of interest not only because of the difficulties associated with this kind of testing, but also because of the potential for solving the problem at hand. It is important that the work continues, and future efforts will concentrate on reducing the error in efficiency prediction to an acceptable margin.

\section{ACKNOWLEDGEMENTS}

The authors wish to acknowledge the National Research Foundation for funding the project.

\section{REFERENCES}

Angle, T. and Crisswell, J. (1997) Envirotech Pump System: Slurry pump manual, Envirotech.

Brito-De la Fuente, E., Leuliet, J.C., Choplin, L. and Tanguy, P. (1992) On the effect of shear-thinning behaviour on mixing with helical ribbon impeller. Chemical and Biochemical Applications, G. Tatterson and R. Calabrese Eds., pp. 28.

Brito-De la Fuente, E., Choplin, L. and Tanguy, P. (1997) Mixing with helical ribbon impeller: Effect of highly Shear Thinning Behaviour and Impeller Geometry. Trans IChemE, Vol 75, Part A.

International Standard 9906 (1999) Rotodynamic pumps - Hydraulic performance acceptance tests - Grades 1 and 2.

Metzner, A.B. and Otto, R.E. (1957) Agitation of non-Newtonian fluids. AIChE J., 3, 3.

Rieger, F. and Novak, V. (1973) Power consumption of agitators in highly viscous non-Newtonian fluids. Chem. Engrs, 51, pp. 10511.

Slatter, P..T. (2005) Tailings transport - back to basics!, Invited keynote address, Paste 2005, International Seminar on Paste and Thickened Tailings, Santiago, Chile 20-22 April, 2005, pp. 165-176.

Walker, C.I. and Goulas, A. (1984) Performance characteristics of centrifugal pumps when handling non-Newtonian slurries. Proc. Inst. Mech. Engrs., 198A(1), pp. 41-49. 